\title{
Indução de puberdade em leitoas com diferentes idades em dois sistemas de manejo
}

\author{
Renato Rosa Ribeiro(1), Diogo Magnabosco(1), Thomas Bierhals(1), Thais Schwarz Gaggini(1), \\ Mari Lourdes Bernardi(2), Fernando Pandolfo Bortolozzo(1) e Ivo Wentz ${ }^{(1)}$
}

(1)Universidade Federal do Rio Grande do Sul (UFRGS), Faculdade de Veterinária, Setor de Suínos, Avenida Bento Gonçalves, no 9.090, CEP 91540-000 Porto Alegre, RS. E-mail: renatorosaribeiro@hotmail.com, diogomagnabosco@yahoo.com.br, thombier@hotmail.com, tsgaggini@yahoo.com.br, fpbortol@ufrgs.br, ivowentz@ufrgs.br (2)UFRGS, Faculdade de Agronomia, Departamento de Zootecnia, Avenida Bento Gonçalves, № 7.712, CEP 91540-000 Porto Alegre, RS. E-mail: mari.bernardi@ufrgs.br

Resumo - O objetivo deste trabalho foi determinar o efeito do método de exposição ao macho e da idade da leitoa, por ocasião da exposição, sobre o percentual de manifestação do primeiro estro, idade de início da puberdade e intervalo entre estímulo e puberdade. Leitoas Danbred F1 Landrace x Large White foram induzidas à puberdade com dois sistemas de exposição ao macho - Baia, estímulo feito pela introdução de macho na baia das fêmeas; Bear, estímulo feito pela colocação de fêmeas na área de permanência de machos -, em três idades de início do estímulo - 150,170 e 200 dias. Não houve diferença entre os sistemas de estimulação quanto à percentagem de leitoas em estro, idade à puberdade e intervalo entre estimulação e puberdade. Esse intervalo foi menor, quando a exposição das fêmeas foi feita aos 200 dias de idade, e a proporção de fêmeas que atingiram a puberdade aos $10,20,30$ e 42 dias após o início da exposição foi maior. A indução da puberdade tem maior sucesso quando a exposição da fêmea ao macho é feita aos 200 dias de idade, independentemente do sistema de estimulação.

Termos para indexação: estimulação à puberdade, idade ao primeiro estro, nulípara.

\section{Puberty induction in gilts at different ages in two management systems}

\begin{abstract}
The objective of this work was to determine the effect of boar-exposure method and of gilt age at exposure on the percentage of first estrus manifestation, puberty age, and interval between stimulus and puberty. Danbred F1 Landrace x Large White gilts were induced to puberty with two different systems of boar exposure - Baia, stimuli done by putting a male inside the pens of gilts; Bear, stimuli done by putting the females in an area with males -, and three ages at the beginning of stimulation - 150, 170 and 200 days. There was no difference between the stimulation systems for the percentage of gilts in estrus, puberty age, and the interval between stimulation and estrus onset. This interval was lower when stimulus started at 200 days, and the proportion of gilts which reached puberty at 10,20,30 and 42 days after the beginning of boar-exposure was higher. Puberty induction has higher success when the female exposure to the male is done at 200 days, regardless of the boar-exposure system.
\end{abstract}

Index terms: stimulation of puberty, age of first estrus, nulliparous.

\section{Introdução}

A suinocultura tecnificada apresenta taxas de reposição de matrizes de até $50 \%$ ao ano (Kummer et al., 2005). Os cuidados com as leitoas antes da primeira cobertura são importantes para a obtenção de desempenho reprodutivo satisfatório ao primeiro parto e ao longo da vida (Bortolozzo et al., 2009). Segundo Wettere et al. (2006), um dos principais cuidados a serem tomados é a redução da variação da idade à puberdade dentro de um grupo de leitoas. Nesse sentido, o manejo da indução da puberdade é praticado pela exposição das leitoas aos machos com idade superior a 10 meses (Kirkwood \& Hughes, 1979) e com alta libido (Hughes,
1994), o que permite um contato focinho com focinho diário com todas as leitoas do grupo.

Aplicando-se esse protocolo, as leitoas atingem a idade da puberdade entre 150 e 220 dias (Tummaruk et al., 2007; Soede et al., 2011). A redução dessa amplitude pode ser obtida pela indução precoce da puberdade, com a maior concentração e sincronização de leitoas em estro em um período de até 30 dias após o início da estimulação das fêmeas. Além de benefícios no desempenho reprodutivo (Kummer et al., 2006; Patterson et al., 2010), a antecipação da idade da puberdade traz benefícios econômicos, uma vez que os dias não produtivos de uma granja podem ser reduzidos pelo acasalamento de leitoas em idade mais precoce (Evans \& O’Doherty, 2001).

Pesq. agropec. bras., Brasília, v.47, n.10, p.1518-1523, out. 2012 
A restrição de espaço, tempo e mão de obra pode determinar o método de exposição ao macho a ser empregado (Patterson et al., 2002). O sistema mais utilizado é o da introdução de um macho na baia das leitoas. O outro sistema consiste em estimular as leitoas numa área específica de exposição ao macho (Siswadi \& Hughes, 1995; Patterson et al., 2002). No manejo tradicional, a estimulação da puberdade de fêmeas é feito com um macho por baia. No sistema com exposição por área, há estímulo simultâneo com vários machos (Hemsworth et al., 1991; Langendijk et al., 2000). A hipótese, nesse caso, é a de que fêmeas estimuladas com esse sistema apresentariam maior manifestação de estro, independentemente da idade do início do manejo de indução, em razão do maior estímulo (contato visual, olfativo, auditivo e físico) oferecido pela presença simultânea (Hemsworth et al., 1991) de vários machos na área de manejo.

A idade de início da puberdade pode variar, pois depende de uma série de fatores, como ganho de peso da fêmea até o estímulo com o macho (Amaral Filha et al., 2009; Kummer et al., 2009), genótipo da leitoa - que também está relacionado ao ganho de peso -, idade em que o estímulo é iniciado (Amaral Filha et al., 2009; Kummer et al., 2009) e qualidade e frequência da realização do estímulo com o macho (Hughes \& Cole, 1976; Kirkwood \& Hughes, 1979). Com relação aos diferentes métodos de exposição ao macho, há poucos estudos que relatam a resposta de indução à puberdade.

O objetivo deste trabalho foi determinar o efeito do método de exposição ao macho e da idade da leitoa, por ocasião da exposição, sobre o percentual de manifestação do primeiro estro, idade de início da puberdade e intervalo entre estímulo e puberdade.

\section{Material e Métodos}

O experimento foi realizado em granja localizada no centro-oeste do Estado de Santa Catarina, Brasil $\left(27^{\circ} 16^{\prime} 58^{\prime \prime} \mathrm{S}, 50^{\circ} 35^{\prime} 04^{\prime \prime} \mathrm{W}\right.$, altitude $\left.987 \mathrm{~m}\right)$, de janeiro a maio de 2010, durante as estações de verão e outono. O clima é caracterizado como subtropical úmido, e a temperatura média no período do experimento variou entre 18,8 e $21,4^{\circ} \mathrm{C}$, enquanto a temperatura mínima variou de 13 a $24,5^{\circ} \mathrm{C}$ e a máxima de 22,0 a $37,5^{\circ} \mathrm{C}$.

$\mathrm{O}$ experimento foi conduzido em granja com plantel de 5.500 matrizes, com sistema de reposição interno.
Ao serem transferidas do setor de recria, as leitoas foram alojadas em baias com $3,80 \times 4,00 \mathrm{~m}$, equipadas com comedouros semiautomáticos e dois bebedouros do tipo chupeta. O manejo alimentar realizado dos 150 aos 180 dias de vida consistiu no fornecimento de ração à vontade - composta de milho e soja com $18,1 \%$ de proteína bruta, 0,98\% de lisina e 3,097 Mcal EM kg-1. Após esse período, as leitoas receberam $3 \mathrm{~kg}$ da mesma ração, fracionados em dois arraçoamentos diários.

No período entre a seleção e o início do estímulo da puberdade, as fêmeas permaneceram sem contato com machos. Durante esse período, as leitoas foram observadas diariamente para verificação da ocorrência de hiperemia e edema vulvar (HEV), além de serem testadas quanto ao reflexo de tolerância ao macho na presença do homem.

Quatrocentos e sessenta e oito leitoas F1 Landrace x Large White (linhagem avó DB 25 - DB-DanBred), com pelo menos seis pares de tetos viáveis, sem problemas de aprumo e casco, foram selecionadas com 150 dias de idade aproximadamente. Foi empregado o delineamento experimental inteiramente casualizado, em esquema fatorial $2 \times 3$, com dois sistemas de manejo para a estimulação da puberdade e três idades de início de exposição ao macho (150, 170 e 200 dias). Um dos sistemas usados para a estimulação foi o convencional (Baia), com o macho conduzido às baias para efetuar o estímulo; o outro foi o da área de exposição ao macho (Bear), em que as fêmeas eram conduzidas a área específica de exposição aos machos (Langendijk et al., 2000; Patterson et al., 2002). Após a seleção, as leitoas foram distribuídas aleatoriamente em cada tratamento, de acordo com suas idades, com grupos de seis baias por tratamento. As fêmeas efetivamente avaliadas ficaram distribuídas nos seis tratamentos da seguinte forma: Baia150 ( $\mathrm{n}=71)$; Bear150 ( $\mathrm{n}=71)$; Baia170 ( $\mathrm{n}=74)$; Bear170 ( $\mathrm{n}=74)$; Baia200 ( $\mathrm{n}=66)$; e Bear200 $(\mathrm{n}=61)$. Não foram submetidas ao estímulo com o macho as leitoas com problemas locomotores e com ganho de peso (GPD) inferior a $580 \mathrm{~g} \mathrm{dia}^{-1}$. Também foram descartadas as leitoas que apresentaram HEV e reflexo de tolerância positivo na presença do homem, antes do início do estímulo com o macho.

As leitoas foram estimuladas por machos sexualmente maduros (idade superior a 12 meses), uma vez ao dia, pelo período de 30 dias. No sistema Baia, o macho foi introduzido na baia das fêmeas e teve contato direto com todo o grupo por um período de 15 
min. No sistema Bear, a área de exposição ao macho possuía duas baias laterais de 3,00×4,60 m e, entre elas, havia quatro gaiolas para o alojamento dos machos. As gaiolas $(2,00 \times 0,55 \mathrm{~m})$ apresentavam dois portões de acesso (um na frente e outro atrás), que facilitavam o acesso dos machos às baias de estimulação e, também, possibilitava o direcionamento desses a ambos os lados, o que permitia o uso simultâneo das duas baias para o contato entre os machos e as leitoas. Nesse sistema, as fêmeas foram conduzidas das baias de alojamento até as baias anexas às gaiolas dos machos. Durante os primeiros $5 \mathrm{~min}$, foi realizada a estimulação focinho a focinho, em que dois machos ficavam de frente para uma baia e os outros dois machos de frente para a outra baia de leitoas. Nos outros 10 min, o grupo de leitoas teve contato físico direto com um dos quatro machos, o qual foi inserido na baia das fêmeas. $\mathrm{O}$ alojamento de quatro machos nas gaiolas possibilitava o seu uso de forma rotativa, em que um macho era utilizado de cada vez para a estimulação das leitoas. Ainda na área central, ao lado das gaiolas dos machos, havia duas gaiolas de apoio. Numa delas, foram alojadas as fêmeas identificadas em estro e, na outra, havia uma balança para a pesagem das fêmeas.

Nos dois sistemas, um técnico treinado auxiliou na estimulação e na condução do macho e realizou o teste da pressão lombar, com intuito de confirmar a manifestação do reflexo de tolerância ao homem na presença do macho. Foram utilizados três grupos de machos para a estimulação, com quatro machos em cada grupo, com boa libido e livres de alterações clínicas. Foi efetuado rodízio diário dos grupos de machos entre os dois sistemas, com um grupo usado no sistema Bear e outro no sistema Baia, enquanto o terceiro grupo permanecia em descanso. Para evitar o cansaço dos machos, cada um deles foi utilizado para estimular no máximo cinco baias por dia.

Fêmeas que apresentaram reflexo de tolerância ao homem na presença do macho (RTM) foram imediatamente retiradas do contato com o macho e transferidas para gaiolas ou baias acessórias. Fêmeas que não apresentaram estro durante os 30 dias de estímulo, em ambos os sistemas, foram transferidas para gaiolas. Nas gaiolas, o estímulo foi realizado por meio da presença do cachaço no corredor em frente, o que possibilitou o contato focinho a focinho com as leitoas. Nessas fêmeas, o diagnóstico de estro foi confirmado pela resposta positiva ao teste de pressão lombar, duas vezes ao dia, durante 12 dias.

$\mathrm{O}$ peso e a espessura de toucinho (ET) das leitoas foram medidos na seleção, no início do período de estímulo com o macho e no momento do estro puberal. A medida da ET foi efetuada no ponto P2, na altura da última costela, $6,5 \mathrm{~cm}$ de distância da linha mediana da coluna vertebral com um aparelho de ultrassonografia Lean-Meater (Renco Corporation, Minneapolis, MN, EUA), com intervalo de $1 \mathrm{~mm}$.

Por ocasião do estímulo com o macho, as leitoas apresentaram as seguintes características em média: $92,2,107,0$ e 133,3 $\mathrm{kg}$ de peso, 0,611, 0,629 e 0,666 kg de GPD, e 10,4, 11,9 e 14,3 mm de ET, para os grupos de 150,170 e 200 dias de idade, respectivamente. Para os sistemas Baia e Bear, as características das leitoas foram, respectivamente, as seguintes: 173,6 e 173,8 dias de idade; 110,5 e 111,1 kg de peso; 0,633 e $0,636 \mathrm{~kg}$ de GPD; e 12,1 e 12,3 mm de ET.

As análises estatísticas foram realizadas com o programa SAS (SAS Institute, 2005). Os percentuais de leitoas que manifestaram estro aos 10, 20, 30 e 42 dias após o início da exposição ao macho foram comparados pelo teste qui-quadrado. Cada leitoa foi considerada uma unidade experimental. As seguintes variáveis foram analisadas pelo procedimento GLM (modelos lineares gerais): peso, GPD e ET, à manifestação do primeiro estro; idade à manifestação do estro; e intervalo entre a exposição ao macho e a manifestação de estro. Nos modelos usados para essas variáveis, foram incluídos os efeitos do sistema de estímulo (Baia ou Bear), da idade no início da exposição ao macho $(150,170$ ou 200 dias) e da interação entre esses dois fatores. Quando o efeito foi significativo, as comparações foram realizadas pelo teste de Tukey-Kramer a 5\% de probabilidade.

\section{Resultados e Discussão}

Não houve efeito do sistema de estimulação e nem da interação entre idade e sistema de estimulação sobre a percentagem de manifestação de estro (Tabela 1). Nos poucos estudos desenvolvidos em sistema com área específica para estimulação, também não foram observadas diferenças na indução de puberdade, em comparação ao sistema convencional de estimulação das fêmeas na baia (Siswadi \& Hughes, 1995; 
Patterson et al., 2002). Porém, em ambos os sistemas de estimulação, um maior percentual de manifestação de estro aos 10, 20, 30 e 42 dias, após o início do estímulo, foi observado nas fêmeas com 200 dias de idade, em comparação com as de 150 e 170 dias de idade, as quais não diferiram entre si.

Em virtude de o sistema Bear proporcionar movimentação das leitoas, poderia haver um maior efeito do macho, em consequência do aumento dos pulsos de hormônio luteinizante ( $\mathrm{LH})$, mediado pelo aumento de cortisol, ocasionado pelo estresse do transporte/realocação (Hughes et al., 1997). Esses autores relataram efeito significativo da interação entre o estresse do transporte e o estímulo com o macho, que acarretou redução da idade ao primeiro estro. Contudo, no experimento de Hughes et al. (1997) o transporte das fêmeas foi feito em um reboque por $20 \mathrm{~min}$, o que poderia ter aumentado o estresse em comparação com o presente trabalho, em que a distância percorrida pelas fêmeas foi de poucos metros. Assim, é possível que o deslocamento até a área de estimulação não tenha sido suficiente para causar o estresse necessário para uma maior resposta por parte das leitoas. Além disso, depois de 2-3 dias, as leitoas se mostraram adaptadas a esse deslocamento, que passou a não constituir um fator estressante.

Tabela 1. Manifestação de estro após diferentes períodos, de acordo com o sistema de estimulação e com a idade das leitoas no início do estímulo com o macho ${ }^{(1)}$.

\begin{tabular}{|c|c|c|c|c|c|}
\hline \multirow[t]{2}{*}{ Idade(dias) } & \multirow[t]{2}{*}{ Número de leitoas } & \multicolumn{4}{|c|}{ Manifestação de estro (\%) } \\
\hline & & 10 dias & 20 dias & 30 dias & 42 dias \\
\hline & & \multicolumn{4}{|c|}{ Sistema Baia } \\
\hline 150 & 71 & $9,9 a$ & $14,1 \mathrm{a}$ & $28,2 \mathrm{a}$ & $46,5 \mathrm{a}$ \\
\hline 170 & 74 & $13,5 \mathrm{a}$ & $25,7 \mathrm{a}$ & $33,8 \mathrm{a}$ & $50,0 \mathrm{a}$ \\
\hline \multirow[t]{2}{*}{200} & 66 & $33,3 b$ & $57,6 \mathrm{~b}$ & $77,3 b$ & $81,8 \mathrm{~b}$ \\
\hline & & \multicolumn{4}{|c|}{ Sistema Bear } \\
\hline 150 & 71 & $12,7 \mathrm{a}$ & $21,1 \mathrm{a}$ & $36,6 \mathrm{a}$ & $47,9 \mathrm{a}$ \\
\hline 170 & 74 & $13,5 \mathrm{a}$ & $28,4 \mathrm{a}$ & $39,2 \mathrm{a}$ & $48,6 \mathrm{a}$ \\
\hline 200 & 61 & $34,4 b$ & $63,9 b$ & $77,0 \mathrm{~b}$ & $78,7 \mathrm{~b}$ \\
\hline
\end{tabular}

${ }^{(1)}$ Médias seguidas por letras iguais nas colunas, dentro de cada sistema, não diferem pelo pelo teste de Tukey-Kramer, a 5\% de probabilidade. Baia, estímulo efetuado pela introdução do macho na baia das fêmeas; Bear, estímulo efetuado pela colocação das fêmeas na área de permanência do macho.
O uso eficiente do manejo em rodízio de machos também pode contribuir para explicar a ausência de diferenças entre os sistemas Bear e Baia. Esse manejo em rodízio possibilitou que todas as leitoas tivessem contato com todos os machos e, caso existisse variação de libido entre estes, o efeito teria influenciado os resultados dos dois sistemas. Além disso, a utilização de três grupos com quatro machos, em sistema de rodízio, permitiu um período de descanso maior aos animais. Castro et al. (1996) sugerem que quando este rodízio não é realizado de forma adequada, os machos podem ficar estressados, o que prejudicaria a libido. Neste caso, o sistema Bear poderia ter apresentado vantagem sobre o sistema Baia, pois a presença de vários machos minimizaria o efeito indesejável de um macho com baixa libido.

O intervalo entre o início do estímulo e a manifestação de estro, a idade de manifestação de estro, o peso, o ganho de peso diário e a espessura de toucinho no primeiro estro após a estimulação não foram influenciados pela interação entre sistemas e idades de estimulação nem pelo sistema de estimulação (Tabela 2). A idade ao primeiro estro, o peso e a espessura de toucinho foram diferentes entre as três idades de estimulação. Fêmeas estimuladas com 200 dias apresentaram menor intervalo entre estímulo e estro, e maior ganho de peso diário do que as estimuladas com 150 e 170 dias.

A idade recomendada para o início da estimulação no sistema Baia é de aproximadamente 160 dias (Hughes \& Cole, 1976), com 70 a $85 \%$ de manifestação de estro em um período de 30 a 40 dias (Amaral Filha et al., 2009; Kummer et al., 2009). Entretanto, há variação de acordo com a genética (Tummaruk et al., 2007), cruzamento utilizado e com o ganho de peso das leitoas (Amaral Filha et al., 2009; Kummer et al., 2009). No presente estudo, menos de $40 \%$ das fêmeas estimuladas a partir de 150 ou 170 dias manifestaram estro até 30 dias após o estímulo com o macho (Tabela 1), o que é considerado como resposta inadequada, se confrontada com os percentuais de $82-84 \%$ obtidos em leitoas estimuladas com idade semelhante, mas da linhagem Camborough 22 - Agroceres PIC (Amaral Filha et al., 2009).

Embora estudos prévios tenham induzido com sucesso a puberdade em fêmeas estimuladas aos 160 dias de idade (Hughes \& Cole, 1976), ou mesmo em idade mais precoce (Kummer et al., 2009), os resultados do presente estudo não corroboraram esses 
resultados, mesmo com uso de genótipos modernos no cruzamento. Vários autores sugerem que animais selecionados para maior deposição de tecido magro podem apresentar maturação reprodutiva mais tardia (Gaughan et al., 1997; Evans \& O’Doherty, 2001; Wettere et al., 2006). É provável que o genótipo usado no presente estudo tenha maturação fisiológica do eixo-hipotálamo-hipófise-gônadas mais tardia do que outros genótipos modernos, o que explicaria os baixos percentuais de manifestação da puberdade observados nas leitoas estimuladas aos 150 e 170 dias de idade.

A necessidade de iniciar a estimulação das leitoas desse genótipo com 200 dias de idade, implica aumento dos dias não produtivos, além do aumento do volume de trabalho, necessidade de mão de obra e de instalações extras para alojar as leitoas, em comparação com início do estímulo aos 150-170 dias de idade. A definição de uma idade ideal para iniciar a estimulação da puberdade do genótipo utilizado neste trabalho, portanto, é fundamental para otimizar a produtividade e evitar manejos desnecessários de estimulação da puberdade em idade precoce. Assim, é importante que se investigue se leitoas com maior ganho de peso apresentariam melhor resposta quando estimuladas em idade mais precoce, conforme mostrado em outros genótipos, com estimulação em idade próxima aos 140 dias (Amaral Filha et al., 2009; Kummer et al., 2009).

Tabela 2. Intervalo entre o início do estímulo e manifestação do estro, idade, peso, ganho de peso diário (GPD) e espessura de toucinho (ET) à puberdade, de acordo com o sistema e idade de estimulação (médias \pm erro padrão) ${ }^{(1)}$.

\begin{tabular}{|c|c|c|c|c|}
\hline \multirow[t]{2}{*}{ Sistema } & \multicolumn{4}{|c|}{ Idade ao início do estímulo com o macho (dias) } \\
\hline & 150 & 170 & 200 & Média \\
\hline & \multicolumn{4}{|c|}{ Intervalo para a manifestação de estro (dias) } \\
\hline Baia & $24,2 \pm 1,9$ & $21,1 \pm 1,8$ & $15,6 \pm 1,5$ & $20,3 \pm 1,0$ \\
\hline Bear & $21,5 \pm 1,9$ & $19,0 \pm 1,9$ & $13,5 \pm 1,6$ & $18,0 \pm 1,0$ \\
\hline \multirow[t]{2}{*}{ Média } & $22,9 \pm 1,4 \mathrm{a}$ & $20,0 \pm 1,3 \mathrm{a}$ & $14,6 \pm 1,1 \mathrm{~b}$ & - \\
\hline & \multicolumn{4}{|c|}{ Idade de manifestação do estro (dias) } \\
\hline Baia & $176,5 \pm 1,9$ & $191,3 \pm 1,8$ & $214,8 \pm 1,5$ & $194,2 \pm 1,0$ \\
\hline Bear & $172,9 \pm 1,9$ & $189,2 \pm 1,9$ & $213,8 \pm 1,6$ & $192,0 \pm 1,0$ \\
\hline \multirow[t]{2}{*}{ Média } & $174 \pm 1,4 \mathrm{a}$ & $190,3 \pm 1,3 b$ & $214 \pm 1,1 \mathrm{c}$ & - \\
\hline & \multicolumn{4}{|c|}{ Peso no momento do estro (kg) } \\
\hline Baia & $110,3 \pm 2,4$ & $121,4 \pm 2,2$ & $145,8 \pm 1,9$ & $125,8 \pm 1,2$ \\
\hline Bear & $110,3 \pm 2,3$ & $121,2 \pm 2,3$ & $143,2 \pm 2,0$ & $124,9 \pm 1,3$ \\
\hline \multirow[t]{2}{*}{ Média } & $110,3 \pm 1,7 \mathrm{a}$ & $121 \pm 1,6 b$ & $144,5 \pm 1,3 \mathrm{c}$ & - \\
\hline & \multicolumn{4}{|c|}{ GPD no momento do estro (kg) } \\
\hline Baia & $0,626 \pm 0,01$ & $0,636 \pm 0,01$ & $0,679 \pm 0,008$ & $0,647 \pm 0,005$ \\
\hline Bear & $0,645 \pm 0,01$ & $0,643 \pm 0,01$ & $0,668 \pm 0,009$ & $0,652 \pm 0,005$ \\
\hline \multirow[t]{2}{*}{ Média } & $0,635 \pm 0,007 \mathrm{a}$ & $0,639 \pm 0,007 \mathrm{a}$ & $0,673 \pm 0,005 b$ & - \\
\hline & \multicolumn{4}{|c|}{ ET no estro (mm) } \\
\hline Baia & $11,7 \pm 0,4$ & $12,4 \pm 0,4$ & $15,8 \pm 0,3$ & $13,3 \pm 0,2$ \\
\hline Bear & $11,7 \pm 0,4$ & $13,3 \pm 0,4$ & $15,1 \pm 0,3$ & $13,3 \pm 0,2$ \\
\hline Média & $11,7 \pm 0,3 \mathrm{a}$ & $12,8 \pm 0,3 \mathrm{~b}$ & $15,5 \pm 0,2 \mathrm{c}$ & - \\
\hline
\end{tabular}

${ }^{(1)}$ Médias seguidas por letras iguais, nas linhas, não diferem, entre si, pelo teste de Tukey-Kramer, a 5\% de probabilidade. Baia, estímulo efetuado pela introdução do macho na baia das fêmeas; Bear, estímulo efetuado pela colocação das fêmeas na área de permanência do macho. 


\section{Conclusões}

1. Para indução da puberdade de leitoas Danbred F1 Landrace $\times$ Large White, o estímulo com o macho deve ser iniciado aos 200 dias de idade.

2. Os sistemas com os estímulos feitos na baia ou em área específica apresentam igual eficácia para a indução da puberdade de leitoas Danbred F1 Landrace $\times$ Large White.

\section{Referências}

AMARAL FILHA, W.S.; BERNARDI, M.L.; WENTZ, I.; BORTOLOZZO, F.P. Growth rate and age at boar exposure as factors influencing gilt puberty. Livestock Science, v.120, p.51-57, 2009

BORTOLOZZO, F.P.; BERNARDI, M.L.; KUMMER, R.; WENTZ, I. Growth, body state and breeding performance in gilts and primiparous sows. Society of Reproduction and Fertility Supplement, v.66, p.281-291, 2009.

CASTRO, M.L.S. de; DESCHAMPS, J.C.; MEINKE, W.; SIEWEDT, F.; CARDELINO, R.A. Influência do período de coleta sobre o volume e doses de sêmen em suínos. Ciência Rural, v.26, p.457-462, 1996.

EVANS, A.C.O.; O'DOHERTY, J.V. Endocrine changes and management factors affecting puberty in gilts. Livestock Production Science, v.68, p.1-12, 2001.

GAUGHAN, J.B.; CAMERON, R.D.A.; DRYDEN, G.M.; YOUNG, B.A. Effect of body composition at selection on reproductive development in large white gilts. Journal of Animal Science, v.75, p.1764-1772, 1997.

HEMSWORTH, P.H.; HANSEN, C.; COLEMAN, G.J.; JONGMAN, E. The influence of conditions at the time of mating on reproduction of commercial pigs. Applied Animal Behaviour Science, v.30, p.273-285, 1991.

HUGHES, P.E. The influence of boar libido on the efficacy of boar effect. Animal Reproduction Science, v.35, p.111-118, 1994.

HUGHES, P.E.; COLE, D.J.A. Reproduction in the gilt. 2. The influence of gilt age at boar introduction on the attainment of puberty. Animal Production, v.23, p.89-94, 1976.

HUGHES, P.E.; PHILIP, G.; SISWADI, R. The effects of contact frequency and transport on the efficacy of the boar effect. Animal Reproduction Science, v.46, p.159-165, 1997.
KIRKWOOD, R.N.; HUGHES, P.E. The influence of age at first boar contact on puberty attainment in the gilt. Animal Production, v.29, p.231-238, 1979.

KUMMER, R.; BERNARDI, M.L.; SCHENKEL, A.C.; AMARAL FILHA, W.S.; WENTZ, I.; BORTOLOZZO, F.P. Reproductive performance of gilts with similar age but with different growth rate at the onset of puberty stimulation. Reproduction in Domestic Animals, v.44, p.255-259, 2009.

KUMMER, R.; BERNARDI, M.L.; WENTZ, I.; BORTOLOZZO, F.P. Reproductive performance of high growth rate gilts inseminated at an early age. Animal Reproduction Science, v.96, p.47-53, 2006.

KUMMER, R.; BORTOLOZZO, F.P.; WENTZ, I.; BERNARDI, M.L. Existe diferença no desempenho reprodutivo ao primeiro parto de leitoas inseminadas no 1을 $2^{\circ}, 3^{\circ}$ ou $4^{\circ}$ estro? Acta Scientiae Veterinariae, v.33, p.125-130, 2005.

LANGENDIJK, P.; SOEDE, N.M.; KEMP, B. Effects of boar contact and housing conditions on estrus expression in weaned sows. Journal of Animal Science, v.78, p.871-878, 2000.

PATTERSON, J.L.; BELTRANENA, E.; FOXCROFT, G. The effect of gilt age at first estrus and breeding on third estrus on sow body weight changes and long-term reproductive performance. Journal of Animal Science, v.88, p.2500-2513, 2010.

PATTERSON, J.L.; WILLIS, H.J.; KIRKWOOD, R.N.; FOXCROFT, G.R. Impact of boar exposure on puberty attainment and breeding outcomes in gilts. Theriogenology, v.57, p.2015-2025, 2002.

SAS INSTITUTE. SAS/STAT user's guide. Version 9.1.3. Cary: SAS Institute, 2005.

SISWADI, R.; HUGHES, P.E. The efficacy of the boar effect when conducted in a modified detection-mating area (DMA). Australian Journal of Agricultural Research, v.46, p.1517-1523, 1995.

SOEDE, N.M.; LANGENDIJK, P.; KEMP, B. Reproductive cycles in pigs. Animal Reproduction Science, v.124, p.251-258, 2011.

TUMMARUK, P.; TANTASUPARUK, W.; TECHAKUMPHU, M.; KUNAVONGKRIT, A. Age, body weight and backfat thickness at first observed oestrus in crossbred Landrace x Yorkshire gilts, seasonal variations and their influence on subsequence reproductive performance. Animal Reproduction Science, v.99, p.167-81, 2007.

WETTERE, H.E.J. van; REVELL, D.K.; MITCHELL, M.; HUGHES, P.E. Increasing the age of gilts at first boar contact improves the timing and synchrony of the pubertal response but does not affect potential litter size. Animal Reproduction Science, v.95, p.97-106, 2006.

Recebido em 1ํㅡe fevereiro de 2012 e aprovado em 25 de setembro de 2012

Pesq. agropec. bras., Brasília, v.47, n.10, p.1518-1523, out. 2012 